\title{
(Quasi-)Injective Extending Gamma Modules
}

\author{
Mehdi S. Abbas \\ Saad Abdulkadhim Al-Saadi \\ Emad Allawi Shallal \\ Department of Mathematics, College of Science, Al-Mustansiriyah University \\ m.abass@uomustansiriyah.edu.iq_s.alsaadi@uomustansiriyah.edu.iq \\ emad_a_shallal@yahoo.com
}

$\begin{array}{lll}\text { Recived : } 14 \backslash 8 \backslash 2017 & \text { Revised : // } & \text { Accepted : 7\9\2017 }\end{array}$

\begin{abstract}
In this paper we introduce and study the concept of injective (Quasi-injective) extending gamma modules as a generalization of injective (Quasi-injective) gamma modules. An $R_{\Gamma}$-module $E$ is called injective (Quasi-injective) extending gamma modules if each proper $R_{\Gamma}$-submodule in $E$ is essential in injective (Quasi-injective) $R_{\Gamma}$-submodule of $E$. The concept of injective extending gamma modules lie between injective gamma modules and quasi-injective gamma modules.
\end{abstract}

Keywords: Gamma module, injective gamma module, quasi-injective gamma module, extending gamma module, essential gamma submodule, closed gamma submodule.

\section{Mathematics subject classification:16D10,16D40,16D50,16D60,16D90.}

\section{Preliminaries:}

Let $R$ and $\Gamma$ be two additive abelian groups, $R$ is called a $\Gamma$-ring (in the sense of Barnes), if there exists a mapping $\cdot: R \times \Gamma \times R \rightarrow R$, written $\cdot(r, \gamma, s) \mapsto r \gamma s$ such that $(a+b) \alpha c=$ $a \alpha c+b \alpha c, a(\alpha+\beta) c=a \alpha c+a \beta c, a \alpha(b+$ $c)=a \alpha b+b \alpha c$ and $(a \alpha b) \beta c=a \alpha(b \beta c)$ for all $a, b, c \in R$ and $\alpha, \beta \in \Gamma$ [4]. A subset $A$ of $\Gamma-\operatorname{ring} R$ is said to be a right (left) ideal of $R$ if $A$ is an additive subgroup of $R$ and $A \Gamma R \subseteq$ $A(R \Gamma A \subseteq A)$, where $A \Gamma R=\{a \alpha r: a \in A, \alpha \in$ $\Gamma, r \in R\}$. If $A$ is both right and left ideal, we say that $A$ is an ideal of . An element 1 in $\Gamma-$ ring $R$ is unity if there exists element $\gamma_{\circ} \in \Gamma$ such that $r=1 \gamma_{\circ} r=r \gamma_{\circ} 1$ for every $r \in R$, in this paper we denote $\gamma_{\circ} \in \Gamma$ to the element such that $1 \gamma_{\circ}$ is the unity, unities in $\Gamma$-rings differ from unities in rings, it is possible for a $\Gamma$-ring have more than one unity [9]. A $\Gamma-\operatorname{ring} R$ is called commutative, if $r \gamma s=s \gamma r$ for any $r, s \in R$ and $\gamma \in \Gamma$.

Let $R$ be a $\Gamma$-ring and $M$ be an additive abelian group. Then $M$ together with a mapping $: R \times \Gamma \times M \rightarrow M$, written . $(r, \gamma, m) \mapsto r \gamma m$ such that $r \alpha\left(m_{1}+m_{2}\right)=$ $r \alpha m_{1}+r \alpha m_{2},\left(r_{1}+r_{2}\right) \alpha m=r_{1} \alpha m+r_{2} \alpha m$, $r(\alpha+\beta) m=r \alpha m+r \beta m, \quad\left(r_{1} \alpha r_{2}\right) \beta m=$ $r_{1} \alpha\left(r_{2} \beta m\right)$ for each $r, r_{1}, r_{2} \in R, \alpha, \beta \in \Gamma$ and $m, m_{1}, m_{2} \in M$, is called a left $R_{\Gamma}$-module, similarly one can defined right $R_{\Gamma}$-module [4]. A left $R_{\Gamma}$-module $M$ is unitary if there exist 
elements, say 1 in $R$ and $\gamma_{\circ} \in \Gamma$ such that $1 \gamma_{\circ} m=m$ for every $m \in M$.

Let $M$ be an $R_{\Gamma}$-module. A nonempty subset $N$ of $M$ is said to be an $R_{\Gamma}$-submodule of $M$ (denoted by $N \leq M$ ) if $N$ is a subgroup of $M$ and $R \Gamma N \subseteq N$, where $R \Gamma N=\{r \alpha n: r \in R$, $\alpha \in \Gamma, n \in N\}$ [4]. An $R_{\Gamma}$-module $M$ is called simple if $R \Gamma M \neq 0$ and the only $R_{\Gamma}$-submodules of $M$ are $M$ and 0 [6], A $\Gamma$-ring $R$ is called simple if $R \Gamma R \neq 0$ and the only ideals of $R$ are $R$ and 0 . If $X$ is a nonempty subset of $M$, then the $R_{\Gamma}$-submodule of $M$ generated by $X$ denoted by $\langle X\rangle$ and $\langle X\rangle=\cap$ $\{N \leq M: X \subseteq N\}, X$ is called the generator of $\langle X\rangle$ and $\langle X\rangle$ is finitely generated if $|X|<\infty$. In particular, if $X=\{x\}$, then $\langle X\rangle$ is called the cyclic $R_{\Gamma}$-submodule of $M$ generated by $x$. $\langle X\rangle=\left\{\sum_{i=1}^{m} n_{i} x_{i}+\sum_{j=1}^{k} r_{j} \gamma_{j} x_{j}: k, m \in\right.$ $\left.\mathbb{N}, n_{i} \in \mathbb{Z}, \gamma_{j} \in \Gamma, r_{j} \in R, x_{i}, x_{j} \in X\right\}$. If $M$ is unitary, then $\langle X\rangle=\left\{\sum_{i=1}^{n} r_{i} \gamma_{i} x_{i}: n \in \mathbb{N}, \gamma_{i} \in\right.$ $\left.\Gamma, r_{i} \in R, x_{i} \in X\right\}$ [4]. An $R_{\Gamma}$-submodule $N$ of $R_{\Gamma}$-module $M$ is called essential (denote by $\left.N \leq_{e} M\right)$ if every nonzero $R_{\Gamma}$-submodule of $M$ has nonzero intersection with $N$, in this case we say that $M$ is an essential extension of $N$, equivalent to, for each nonzero element $m$ in $M$ there is $r_{1}, r_{2}, \ldots, r_{n} \in R$ and $\gamma_{1}, \gamma_{2}, \ldots, \gamma_{n} \in \Gamma$ such that $\sum_{i=1}^{n} r_{i} \gamma_{i} m(\neq 0) \in N \quad$ [1]. An $R_{\Gamma}$-submodule $N$ of $R_{\Gamma}$-module $M$ is called direct summand of $M$ if there exists an $R_{\Gamma}$-submodule $K$ of $M$ such that $M=N+K$ and $N \cap K=0$, in this case $M$ is written as $M=N \oplus K$ [2]. An $R_{\Gamma}$-module $M$ is called semisimple if every $R_{\Gamma}$-submodule is a direct summand of $M$ [3]. An $R_{\Gamma}$-submodule $N$ of $R_{\Gamma}$-module $M$ is called closed in $M$ if it has no proper essential extension in $M$, equivalent to saying that the only solution of the relation
Mehdi .S / Saad.A / Emad.A

$N \leq_{e} K \leq M$ is $N=K[2]$.

Let $M$ and $N$ be two $R_{\Gamma}$-modules. A mapping $f: M \rightarrow N$ is called homomorphism of $R_{\Gamma}$-modules (simply $R_{\Gamma}$-homomorphism) if $f(x+y)=f(x)+f(y)$ and $f(r \gamma x)=r \gamma f(x)$ for each $x, y \in M, r \in R$ and $\gamma \in \Gamma$. An $R_{\Gamma}$-homomorphism is $R_{\Gamma}$-monomorphism if it is one-to-one and $R_{\Gamma}$-epimorphism if it is onto, the set of all $R_{\Gamma}$-homomorphisms from $M$ into $N$ denote by $\operatorname{Hom}_{R_{\Gamma}}(M, N)$ in particular if $M=N, \operatorname{Hom}_{R_{\Gamma}}(M, N)$ denote by $\operatorname{End}_{R_{\Gamma}}(M)$. If $M$ is $R_{\Gamma}$-module, then $\operatorname{End}_{R_{\Gamma}}(M)$ is a $\Gamma$-ring with the mapping $\because \operatorname{End}_{R_{\Gamma}}(M) \times \Gamma \times$ $\operatorname{End}_{R_{\Gamma}}(M) \rightarrow \operatorname{End}_{R_{\Gamma}}(M)$ denoted by . $(f, \gamma, g) \mapsto f \gamma g$ where $f \gamma g(x)=g(f(1 \gamma x))$, for $f, g \in \operatorname{End}_{R_{\Gamma}}(M), \gamma \in \Gamma$ and $x \in M$. All modules in this paper are unitary left $R_{\Gamma}$-modules, in this case $M$ is a right $\operatorname{End}_{R_{\Gamma}}(M)$-module with the mapping $\because M \times$ $\Gamma \times \operatorname{End}_{R_{\Gamma}}(M) \rightarrow \operatorname{End}_{R_{\Gamma}}(M)$ by $\cdot(x, \gamma, f) \mapsto$ $x \gamma f$ where $x \gamma f=f(1 \gamma x)$, for $f \in \operatorname{End}_{R_{\Gamma}}(M)$, $\gamma \in \Gamma$ and $x \in M[4]$.

The notions of injective gamma modules and quasi-injective gamma modules have been introduced by M. S. Abbas, S. A. Al-saadi and E. A. Shallal in [1] and [2]. If $M$ and $N$ are two $R_{\Gamma}$-modules, then $M$ is called $N$-injective $R_{\Gamma}$-module if for any $R_{\Gamma}$-submodule $A$ of $N$ and for any $R_{\Gamma}$-homomorphism $f: A \rightarrow M$ there exists an $R_{\Gamma}$-homomorphism $g: N \rightarrow M$ such that $g i=f$ where $i$ is the inclusion mapping [1]. An $R_{\Gamma}-$ module $M$ is injective if it is $\quad N$-injective for any $R_{\Gamma}$-module $N$. It is proved in [1], that every gamma module can be embedded in injective gamma module called injective hull and denote by $E(M)$ which is unique up to isomorphism. 


\section{Injective Extending Gamma Modules}

We extended the concept of injective extending gamma modules from category of modules [8] to the category of gamma modules which is lie between injective gamma modules [1] and quasi-injective gamma modules [2].

An $R$-module $M$ is called extending if every submodule of $M$ is essential in a direct summand of $M[8]$.

Definition 2.1. An $R_{\Gamma}$-module $M$ is called $\Gamma$-Extending if every $R_{\Gamma}$-submodule of $M$ is essential in a direct summand of $M$.

Proposition 2.2. An $R_{\Gamma}$-module $M$ is $\Gamma$-Extending if and only if each closed $R_{\Gamma}$-submodule of $M$ is a direct summand of $M$.

Proof: Let $N$ be closed $R_{\Gamma}$-submodule of $\Gamma$-Extending $R_{\Gamma}$-module $M$, then there is an $R_{\Gamma}$-submodule $K$ of $M$ such that $N \leq_{e} K \leq_{\oplus} M$, so $N=K$. Conversely, Let $N$ be a $R_{\Gamma}$-submodule $M$, then by using Zorn's lemma $N$ has a maximal essential extension $K$ in $M$ which is closed, so by hypothesis $K$ is direct summand of $M$, thus $M$ is $\Gamma$-Extending.

The following proposition follows from Corollary(3.11) in [2].

Proposition 2.3. Every quasi-injective $R_{\Gamma}$-module is $\Gamma$-Extending.

The converse of Proposition(2.3) is not true in general as in Example(2.4)(2).

\section{Examples 2.4.}

1- Every semisimple (simple) $R_{\Gamma}$-module is $\Gamma$-Extending. Since every $R_{\Gamma}$-submodule of $M$ is a direct summand, then $M$ is
Mehdi .S / Saad.A / Emad.A

$\Gamma$-Extending. In particular, $Z_{2}$ as $Z_{Z}$-module is $\Gamma$-Extending.

2- Let $\quad R=\{(n n), n \in Z\} \quad$ and $\quad \Gamma=$ $\left\{\left(\begin{array}{l}\gamma \\ 0\end{array}\right), \gamma \in Z\right\}$. Then $R \quad$ is $\Gamma$-ring by $\because R \times \Gamma \times R \rightarrow R$ with $\left(\begin{array}{ll}n & n\end{array}\right)\left(\begin{array}{l}\gamma \\ 0\end{array}\right)\left(\begin{array}{ll}m & m\end{array}\right)=$ $(n \gamma m n \gamma m)$. Let $I \neq 0$ be an ideal of $R$, any another ideal $P$ of $R$ with $P \cap I=0$, take $\quad 0 \neq\left(\begin{array}{ll}n & n\end{array}\right) \in I, \quad 0 \neq\left(\begin{array}{l}\gamma \\ 0\end{array}\right) \in \Gamma \quad$ and $\left(\begin{array}{ll}m & m\end{array}\right)$ any element in $P$, then $\left(\begin{array}{ll}n \gamma m & n \gamma m\end{array}\right)=\left(\begin{array}{ll}n & n\end{array}\right)\left(\begin{array}{l}\gamma \\ 0\end{array}\right)\left(\begin{array}{ll}m & m\end{array}\right) \in R \Gamma P$ $\subseteq P, \quad$ also $\quad(n \gamma m \quad n \gamma m)=$ $\left(\begin{array}{ll}m & m\end{array}\right)\left(\begin{array}{l}\gamma \\ 0\end{array}\right)\left(\begin{array}{ll}n & n\end{array}\right) \in R \Gamma I \subseteq I$, so $n \gamma m=0$, hence $m=0$, thus $P=0$, so every ideal in $R$ is essential, therefore $R$ is $\Gamma$-Extending. Note that $R$ is not quasi-injective, take the ideal $I=\left\{\left(\begin{array}{ll}2 n & 2 n\end{array}\right): n \in Z\right\} \quad$ and $R_{\Gamma}$-homomorphism $\quad \lambda: I \rightarrow R \quad$ by $\lambda\left(\begin{array}{ll}2 n & 2 n\end{array}\right)=\left(\begin{array}{ll}n & n\end{array}\right)$ for each $\left(\begin{array}{ll}2 n & 2 n\end{array}\right) \in$ $I$, if $R$ quasi-injective, then there is $g: R \rightarrow$ $R$ which extends $\lambda$, so $\left(\begin{array}{ll}1 & 1\end{array}\right)=\lambda\left(\begin{array}{ll}2 & 2\end{array}\right)=$ $g\left(\begin{array}{ll}2 & 2\end{array}\right)=g\left(\left(\begin{array}{ll}2 & 2\end{array}\right)\left(\begin{array}{l}1 \\ 0\end{array}\right)\left(\begin{array}{ll}1 & 1\end{array}\right)\right)=$ $\left(\begin{array}{ll}2 & 2\end{array}\right)\left(\begin{array}{l}1 \\ 0\end{array}\right) g\left(\begin{array}{ll}1 & 1\end{array}\right)$, hence $g\left(\begin{array}{ll}1 & 1\end{array}\right)=$ $\left(\frac{1}{2} \frac{1}{2}\right)$ contradiction.

Definition 2.5. An $R_{\Gamma}$-module $M$ is called injective extending $(I \Gamma-$ Extending) if each proper $R_{\Gamma}$-submodule of $M$ is essential in injective $R_{\Gamma}$-submodule of $M$, that is, for each proper $R_{\Gamma}$-submodule $N \leq M$, there exists an injective $R_{\Gamma}$-submodule $K$ of $M$ such that $N \leq_{e} K$.

Proposition 2.6. If $M$ is $I \Gamma$-Extending $R_{\Gamma}$-module, then each proper closed $R_{\Gamma}$-submodule of $M$ is injective. 
Proof: Assume $N \leq M$ is proper closed $R_{\Gamma}$-submodule, then there is an injective $R_{\Gamma}$-submodule $K$ of with $N \leq_{e} K \leq M$, so $N=K$, thus $N$ is injective.

The converse of Proposition(2.3) is not true in general, for example $Z$ as $Z_{Z}$-module, the only proper closed $Z_{Z}-$ submodule of $Z$ which is injective is 0 but $Z$ is not $I \Gamma-$ Extending.

The following proposition gives the converse of Proposition(2.6) under certain conditions. First we note that a semisimple $R_{\Gamma}$-module different from $I \Gamma-$ Extending. The $Z_{Z}-$ module $Z_{2} \oplus Z_{2}$ is semisimple but not $I \Gamma$-Extending, if not, then $Z_{2}$ is injective by Proposition(2.6) contradiction. The $Z_{Z}$-module $Q$ is injective, so $I \Gamma$-Extending but not semisimple.

Proposition 2.7. Let $M \quad$ semisimple $R_{\Gamma}$-module. Then $M$ is a $I \Gamma-$ Extending if and only if each proper closed $R_{\Gamma}$-submodule in $M$ is injective.

Proof: For each proper $R_{\Gamma}$-submodule $N$ of $M, N$ has a maximal essential extension $K$ by Zorn's lemma. It is clear that $K$ is closed and proper, so by hypothesis, $K$ is injective, thus $M$ is $I \Gamma$-Extending.

Proposition 2.8. Let $M$ be $R_{\Gamma}$-module, if $M$ has a proper nonessential $R_{\Gamma}$-submodule, than $M$ is $I \Gamma-$ Extending if and only if each proper closed $R_{\Gamma}$-submodule is injective.

Proof: Assume $N$ is a proper nonessential $R_{\Gamma}$-submodule of an $R_{\Gamma}$-module $M$, then by Zorn's lemma there is maximal $R_{\Gamma}$-submodule $K$ of $M$ such that $N \leq_{e} K$, clear $K$ is closed and proper, so by hypothesis $K$ is injective. By Proposition(1.9) in [3], there is nonzero $R_{\Gamma}$-submodule $L$ of $M$ such that $M=K \oplus L$, again by hypothesis $L$ is injective, hence $M$ is injective[1]. The obverse by Proposition(2.6).
Mehdi .S / Saad.A / Emad.A

Proposition 2.9. If $M$ is $I \Gamma$-Extending $R_{\Gamma}$-module, then every proper closed $R_{\Gamma}$-submodule of $M$ is a direct summand of $M$. In particular, every $I \Gamma$-Extending $R_{\Gamma}$-module is $\Gamma$-Extending.

Proof: Let $N$ is a proper closed $R_{\Gamma}$-submodule of $I \Gamma$-Extending $R_{\Gamma}$-module $M$, then $N$ is injective $R_{\Gamma}$-submodule of $M$ by Proposition(2.6), by Proposition(1.9) in [1] $N$ is a direct summand of $M$.

Proposition 2.10. Every injective $R_{\Gamma}$-module is $I \Gamma$-Extending.

Proof: Let $N$ be a proper $R_{\Gamma}$-submodule of an injective $R_{\Gamma}$-module $M$. Then by Zorn's lemma $N$ has a maximal essential extension $K$ in $M$, clearly that $K$ is closed, by Corollary(3.11) in [2] $K$ is a direct summand in $M$, so $K$ is injective by Examples and Remarks(1.10) (3) in [1], thus $M$ is $I \Gamma$-Extending.

Proposition(2.10) shows that there are a lot of $I \Gamma$-Extending $R_{\Gamma}$-modules, for any $R_{\Gamma}$-module $M$ it's injective hull is $I \Gamma$-Extending . In fact every $R_{\Gamma}$-module can be embedded in $I \Gamma$-Extending $R_{\Gamma}$-module see [1].

Proposition 2.11. Let $M$ be $I \Gamma$-Extending $R_{\Gamma}$-module. If $M$ has a nontrivial closed $R_{\Gamma}$-submodule, then $M$ is injective.

Proof: Let $N$ be a nontrivial closed $R_{\Gamma}$-submodule of $I \Gamma$-Extending $R_{\Gamma}$ - module $M$. Then by Proposition(2.6) $N$ is injective $R_{\Gamma}$-submodule and by Proposition(1.9) in [1] $N$ is a direct summand of $M$, so $M=N \oplus K$ for some $R_{\Gamma}$-submodule $K$ of $M$, since $N$ is a nontrivial, then $K$ is proper closed and again by 
Proposition(2.6) $K$ is injective and so $M$ is injective [1].

\section{Examples 2.12.}

1- Every simple $\quad R_{\Gamma}$-module is $I \Gamma$-Extending, since the only proper closed $R_{\Gamma}$-submodule is 0 .In particular, the $Z_{Z}$-module $\quad M=Z_{2}$ is $I \Gamma$-Extending. Note that $M$ is not injective [1], so the converse of Proposition(2.10) is not true in general.

2- Let $R=Z, \Gamma=Z$ and $M=Z$, then $M$ is not $I \Gamma$-Extending, since the $R_{\Gamma}$-submodule $\langle 2\rangle$ is not essential in any injective $R_{\Gamma}$-submodule. Note that $M$ is a $\Gamma$-Extending since the only closed $R_{\Gamma}$-submodule of $M$ is 0 which is direct summand, hence the converse of Proposition(2.9) is not true in general.

3- Let $M=Z_{6}$ as $Z_{Z}-$ module, the only ideals of $Z_{6}$ are $0, Z_{6},\langle 2\rangle$ and $\langle 3\rangle$, then $Z_{6}$ is semisimple $Z_{Z}$-module but not $I \Gamma-$ Extending.

4- If $R$ is semisimple $R_{\Gamma}$-module, then every $R_{\Gamma}$-module is injective [3], so $I \Gamma-$ Extending.

Direct sum of two $I \Gamma$-Extending $R_{\Gamma}$-modules may not be $I \Gamma-$ Extending, for example The $Z_{Z}$-module $Z_{2}$ is $I \Gamma$-Extending but not injective Examples(2.12)(1), the $Z_{Z}$-module $Z_{2} \oplus Z_{2}$ is not $I \Gamma-$ Extending, if not, then $Z_{2}$ is injective by Proposition(2.6) which is a contradiction .

Proposition 2.13. If direct sum of every two $I \Gamma$-Extending $R_{\Gamma}$-modules is $I \Gamma-$ Extending, then $M$ is injective if and only if $M$ is $I \Gamma-$ Extending.
Mehdi .S / Saad.A / Emad.A

Proof: Let $M$ be $I \Gamma$-Extending, since $E=E(M)$ is injective, so $E$ is $I \Gamma$-Extending by Proposition(2.10), hence by hypothesis $M \oplus E$ is $I \Gamma-$ Extending, by Proposition(2.6) $M$ is injective.

The following proposition gives the converse of Proposition(2.6) under another condition.

Proposition 2.14. Let $M$ be $R_{\Gamma}$-module contains a nontrivial nonessential $R_{\Gamma}$-submodule. Then the following statements are equivalent:

1- $M$ is injective.

2- $\quad M$ is $I \Gamma-$ Extending.

3- Every proper closed $R_{\Gamma}$-submodule of $M$ is injective.

Proof: $\quad(1) \Rightarrow(2) \quad$ By $\quad$ Proposition(2.10). (2) $\Rightarrow$ (3) By Proposition(2.6). (3) $\Rightarrow$ (1) Assume $N$ is a nontrivial $R_{\Gamma}-$ submodule which is not essential in $M$, then by Zorn's lemma $N$ has a maximal essential extension $R_{\Gamma}$-submodule $K$ in $M$ which is closed in $M$, if $K=M$ then $N$ is essential in $M$ contradiction, so $K$ is a proper by hypothesis $K$ is injective and a direct summand of $M$ by Proposition(1.9) in [1], so $M=K \oplus L$ for some $R_{\Gamma}$-submodule $L$ of $M$, if $L=0$ a contradiction, hence $L$ is a proper again by hypothesis $L$ is injective, therefore $M$ is injective [1].

Proposition 2.15. An $R_{\Gamma}$-module $M$ is $I \Gamma$-Extending if and only if $M$ contains injective hulls of each of its proper $R_{\Gamma}$-submodule.

Proof: For each proper $R_{\Gamma}$-submodule $N$ of $I \Gamma$-Extending $R_{\Gamma}$-module $M$, there exists injective $R_{\Gamma}$-submodule $K$ of $M$ such that $N \leq{ }_{e} K \leq M$ but $N \leq E(N)$ which is minimal 
injective extension of $N$, so $N \leq E(N) \leq K \leq$ $M$, hence $E(N) \leq M$.

Corollary 2.16. If $M$ is $I \Gamma$-Extending, then $M$ is injective hull of each proper essential $R_{\Gamma}$-submodule of $M$.

Proof: For each proper essential $R_{\Gamma}$-submodule $N$ of $M$, by Proposition(2.15) $E(N) \leq M$ but $E(N)$ is injective, therefore by Proposition(1.9) in [1] $E(N)$ is a direct summand of $M$, so $M=E(N) \oplus L$ for some $R_{\Gamma}$-submodule $L$ of $M$. But $N \leq_{e} M$, then $L=0$, thus $M=E(N)$.

In the following proposition we a characterization of $I \Gamma$-Extending $R_{\Gamma}$-modules in which every proper $R_{\Gamma}$-submodule lies under injective direct summand.

Proposition 2.17. An $\mathrm{R}_{\Gamma}$-module $M$ is $I \Gamma$-Extending if and only if for every proper $\mathrm{R}_{\Gamma}$-submodule $N$ of $M$, there exists a decomposition $M=M_{1} \oplus M_{2}$ such that $M_{1}$ is injective, $N \leq_{e} M_{1}$ and $N \oplus M_{2} \leq_{e} M$.

Proof: Assume that $N$ is a proper $R_{\Gamma}$-submodule of $M$, then there exists injective $R_{\Gamma}$-submodule $M_{1}$ such that $N \leq_{e} M_{1} \leq M$, so by Proposition(1.9) in [1] $M_{1}$ is a direct summand of $M$, hence $M=M_{1} \oplus M_{2}$ for some $R_{\Gamma}$-submodule $M_{2}$ of $M$, since $M_{2} \leq_{e} M_{2}$ by Lemma(3.3) in [1] $N \oplus M_{2} \leq_{e} M_{1} \oplus M_{2}=M$.

Proposition 2.18. Let $M$ be $R_{\Gamma}$-module. Then $M$ is $I \Gamma-$ Extending if and only if either $M$ is simple or $M$ is injective.

Proof: Assume $M$ is not simple, then there exists a nonzero $R_{\Gamma}$-submodule $N$ of $M$ with $M \neq N$, Also there exists injective $R_{\Gamma}$-submodule $K$ such that $N \leq_{e} K \leq M$ but $K$
Mehdi .S / Saad.A / Emad.A

is a direct summand of $M$ by Proposition(1.9) in [1], then $M=K \oplus L$ for some $R_{\Gamma}$-submodule $L$ of $M$, if $L=M$, then $M$ is injective, if $L \neq M$, then $L$ is proper closed, so by Proposition(2.6) $L$ is injective, hence $M$ is injective [1]. The other direction follows from Proposition(2.10).

Corollary 2.19. Every $I \Gamma$-Extending $R_{\Gamma}$-module is quasi-injective.

Corollary 2.20. Let $M$ be a not simple $R_{\Gamma}$-module. Then $M$ is injective if and only if $M$ is $I \Gamma-$ Extending.

\section{Quasi-Injective Extending Gamma Modules}

In this section we introduce the concept of quasi-injective extending gamma modules as a generalization of quasi-injective gamma modules. An $R_{\Gamma}$-module $M$ is quasi-injective if it is $M$-injective, that is for any $R_{\Gamma}$-submodule $\quad N \quad$ of $\quad M \quad$ and $R_{\Gamma}$-homomorphism $f: N \rightarrow M$, there exists an $R_{\Gamma}$-endomorphism $g$ of $M$ such that $g i=f$ where $i$ is the inclusion mapping of $N$ into $M$ [2].

Definition 3.1. An $R_{\Gamma}$-module $M$ is called quasi-injective extending gamma (simply $Q I \Gamma$-Extending) if every proper $R_{\Gamma}$-submodule of $M$ is essential in a quasiinjective $R_{\Gamma}$-submodule of $M$, that is, for each proper $R_{\Gamma}$-submodule $N$ of $M$, there exists an quasi-injective $R_{\Gamma}$-submodule $K$ of $M$ such that $N \leq_{e} K$.

Proposition 3.2. If $M$ is $Q I \Gamma$-Extending $R_{\Gamma}$-module, then each proper closed $R_{\Gamma}$-submodule of $M$ is quasi-injective. 
Proof: Assume $N$ is proper closed $R_{\Gamma}$-submodule of $M$, then there exists an quasi-injective $R_{\Gamma}$-submodule $K$ of such that $N \leq_{e} K \leq M$, since $N$ is closed then $N=K$.

The converse of Proposition(3.2) is not true in general, for example $M=Z$ as $Z_{Z}$-module.

Proposition 3.3. If $M$ is $Q I \Gamma$-Extending $R_{\Gamma}$-module, then $N \cap M$ is quasi-injective for each proper direct summand $N$ of $E(M)$.

Proof: Let $N$ be a proper direct summand of $E(M)$, then $E(M)=N \oplus B$ for some $R_{\Gamma}$-submodule $B$ of $E(M)$, we claim that $N \cap M$ is closed in $M$, assume that $N \cap M \leq_{e} K$ where $K$ is an $R_{\Gamma}$-submodule of $M$ with $N \cap M \neq K$, let $k \in K$, then $k=n+b$ where $n \in N$ and $b \in B$. Now consider $k \notin N$, then $b \neq 0$. But $M \leq_{e} E(M)$ and $0 \neq b \in B \leq$ $E(M)$, therefore there is $r_{1}, r_{2}, \ldots, r_{n} \in R$ and $\gamma_{1}, \gamma_{2}, \ldots, \gamma_{n} \in \Gamma$ such that $\sum_{i=1}^{n} r_{i} \gamma_{i} b(\neq 0) \in$ $M$, so $\sum_{i=1}^{n} r_{i} \gamma_{i} k=\sum_{i=1}^{n} r_{i} \gamma_{i} n+\sum_{i=1}^{n} r_{i} \gamma_{i} b$, and $\sum_{i=1}^{n} r_{i} \gamma_{i} n=\sum_{i=1}^{n} r_{i} \gamma_{i} k-\sum_{i=1}^{n} r_{i} \gamma_{i} b \in N \cap M \leq$ $K$, thus $\sum_{i=1}^{n} r_{i} \gamma_{i} b=\sum_{i=1}^{n} r_{i} \gamma_{i} k-\sum_{i=1}^{n} r_{i} \gamma_{i} n \in$ $B \cap K, \quad$ since $(N \cap M) \cap B=0$, then $(N \cap M) \cap(B \cap K)=0$, but $N \cap M \leq_{e} K, \quad$ so $B \cap K=0$, hence $\sum_{i=1}^{n} r_{i} \gamma_{i} b=0$ which is a contradiction, thus $N \cap M$ is closed in $M$, if $N \cap M=M$, then $M \leq N$, so $M \cap B=0$ but $M \leq_{e} E(M)$, then $B=0$ which is a contradiction, thus $N \cap M$ is a proper closed $R_{\Gamma}$-submodule of $M$, so by Proposition(3.2) $N \cap M$ is a quasi-injective.

Proposition 3.4. Every quasi-injective $R_{\Gamma}$-module is $Q I \Gamma-$ Extending.

Proof: Assume $M$ is quasi-injective and $N$ is a proper $R_{\Gamma}$-submodule of $M$, then by Zorn's lemma $N$ is essential in a maximal closed $R_{\Gamma}$-submodule $K$ of $M$, by Corollary(3.11) in
[2] $K$ is a quasi-injective, hence $M$ is $Q I \Gamma-$ Extending.

The converse of Proposition(3.4) is not true in general, see Example(3.8)(5).

Corollary 3.5. Every semisimple $R_{\Gamma}$-module is $Q I \Gamma-$ Extending.

The converse of Corollary(3.5) is not true in general, for Example $M=Q$ as $Z_{Z}$-module is injective, so $Q I \Gamma$-Extending but not semisimple.

An $R_{\Gamma}$-module $M$ is called regular if for each $m \in M$, there exists $f \in \operatorname{Hom}_{R_{\Gamma}}(M, R)$ and $\gamma \in \Gamma$ such that $m=f(m) \gamma m$ [3]. Every cyclic $R_{\Gamma}$-submodule of regular $R_{\Gamma}$-module is a direct summand [3].

Corollary 3.6. Every regular cyclic $R_{\Gamma}$-module is $Q I \Gamma-$ Extending.

The converse of Corollary(3.6) is not true in general, for example $M=Q$ as $Z_{Z}$-module is $Q I \Gamma-$ Extending but not regular.

It is proved in [2], that every gamma module has quasi-injective extension say quasi-injective hull (denote by $Q(M)$ ) which is unique up to isomorphism.

Corollary 3.7. Every $R_{\Gamma}$-module can be embedded in $Q I \Gamma-$ Extending.

\section{Examples 3.8.}

1- If $M$ is $Q I \Gamma-$ Extending, then $M$ contains quasi-injective hull of each it's proper $R_{\Gamma}$-submodules, the proof is essentially as in Proposition(2.15). 
2- An $R_{\Gamma}$-module $M=Z_{6}$ as $Z_{Z}$-module is a semisimple [3], so $M$ is $Q I \Gamma$-Extending by Corollary(3.5). Note that $M$ is not $I \Gamma$-Extending

3- Every $I \Gamma$-Extending is $Q I \Gamma-$ Extending. The converse is not true for example see Example(2).

4- The $Z_{Z}$-module $\quad M=Z \oplus Z_{2}$ is not $Q I \Gamma-$ Extending, if not the $R_{\Gamma}$-submodule $N=Z \oplus(0)$ is proper closed in $M$ which is not quasi-injective [2], which is a contradiction by Proposition(3.2).

5- Let $M=Z_{2} \oplus Z_{4}$ as $Z_{Z}$ - module. The only $R_{\Gamma}$-submodules of $M$ are (0), $N_{1}=$ $(0) \oplus Z_{4}, \quad N_{2}=Z_{2} \oplus(0), \quad N_{3}=Z_{2} \oplus\langle 2\rangle=$ $\{(0,0),(1,2),(1,0),(0,2)\}, \quad N_{4}=\langle(1,1)\rangle=$ $\{(0,0),(1,1),(0,2),(1,3)\}, \quad N_{5}=\langle(0,2)\rangle=$ $\{(0,0),(0,2)\}, N_{6}=\langle(1,2)\rangle=\{(0,0),(1,2)\}$ and $M$. Note that $N_{2}, N_{5}$ and $N_{6}$ are simple, $N_{3}$ semisimple, $N_{4} \cong N_{1}$, so every proper $R_{\Gamma}$-submodule of $M$ is quasi-injective, hence $M$ is $Q I \Gamma$-Extending but $M$ is not

Mehdi .S / Saad.A / Emad.A quasi-injective since the $R_{\Gamma}$-submodule $N_{5} \cong N_{2}$ but $N_{2}$ is a direct summand while $N_{5}$ is not direct summand which is a contradiction see Corollary(3.9) in [2].

6- The $Z_{Z}$-module $M=Z_{6} \oplus Z_{4}$ is not $Q I \Gamma$-Extending, since $M=N \oplus B$ where $N=\langle 3\rangle \oplus Z_{4}$ and $B=\langle 2\rangle \oplus(0)$, so $N$ is a proper closed of $M$ but $N$ is not quasiinjective since the $R_{\Gamma}$-submodule $K=$ $(0) \oplus\langle 2\rangle \cong Z_{3} \oplus(0)$ of $N$ is not direct summand of $N$ which is contradiction see Corollary(3.9) in [2].

The concept quasi-injective extending gamma modules is a proper generalization of quasiinjective gamma modules, see Examples(3.8)(5).

We conclude from Proposition(2.10), Corollary(2.19) and Proposition(3.4) the following chart of implications for $R_{\Gamma}$-modules

\section{$Q I \Gamma-$ Extending}

\section{Injective $\Rightarrow I \Gamma-$ Extending $\Rightarrow$ Quasi-Injective \\ $\nabla^{\Downarrow}-$ Extending}

An $R_{\Gamma}$-submodule of $Q I \Gamma$-Extending need not be $Q I \Gamma$-Extending, for example The $Z_{Z}$-module $Q$ is injective [1] hence $Q I \Gamma-$ Extending but the $R_{\Gamma}$-submodule $Z$ is not $Q I \Gamma$-Extending by Examples(3.8)(1).

An $R_{\Gamma}$-submodule $N$ of $R_{\Gamma}$-module $M$ is called $R_{\Gamma}$-idempotent if $N=\left(N:_{R_{\Gamma}} M\right) \Gamma N$ and $M$ is called fully $R_{\Gamma}$-idempotent if every $R_{\Gamma}$-submodule of $M$ is $R_{\Gamma}$-idempotent [3].
Proposition 3.9. If $M$ is $Q I \Gamma-$ Extending. Then every proper $R_{\Gamma}$-idempotent $R_{\Gamma}$-submodule of $M$ is quasi-injective ( $Q I \Gamma-$ Extending).

Proof: Let $N$ be a proper $R_{\Gamma}$-idempotent $R_{\Gamma}$-submodule of $Q I \Gamma-$ Extending $M$. Then $M$ contains quasi-injective hull $Q(N)$ of $N$ by Examples(3.8). Since $N$ is $R_{\Gamma}$-idempotent, then $\quad N=\left(N:_{R_{\Gamma}} Q(N)\right) \Gamma N$. For each $R_{\Gamma}$-submodule $\quad X \quad$ of $N \quad$ and $R_{\Gamma}$-homomorphism $f: X \rightarrow N$, there exists $g: Q(N) \rightarrow Q(N)$ which extends $f$, for each $n \in N, \quad n=r \gamma m$ where $\quad r \in\left(N:_{R_{\Gamma}} Q(N)\right)$, 
$\gamma \in \Gamma \quad$ and $\quad m \in N$, so $g(n)=g(r \gamma m)=$ $\operatorname{r\gamma g}(m) \in N$, thus $N$ is quasi-injective [3].

Corollary 3.10. Let $M$ be fully $R_{\Gamma}$-idempotent. Then $M$ is $Q I \Gamma$-Extending if and only if every $R_{\Gamma}$-submodule of $M$ is $Q I \Gamma-$ Extending.

An $R_{\Gamma}$-module $M$ is called duo if $f(N) \subseteq$ $N$ for each $R_{\Gamma}$-submodule $N$ of $M$ and $f \in \operatorname{End}_{R_{\Gamma}}(M)[3]$.

Corollary 3.11. If $M$ is duo $I \Gamma$-Extending $R_{\Gamma}$-module, then every $R_{\Gamma}$-submodule of $M$ is quasi-injective.

Proof: By Corollary(2.19) $M$ is quasi-injective. For any proper $R_{\Gamma}$-submodule $N$ of $M$, let $X$ be an $R_{\Gamma}$-submodule of $N$ and $f: X \rightarrow N$ be an $R_{\Gamma}$-homomorphism, then there exists $\alpha: E(M) \rightarrow E(M)$ which extends to $f$, since $M$ is quasi-injective, then $\alpha(M) \subseteq M$ [2], hence $\theta=\alpha_{\left.\right|_{M}}: M \rightarrow M$ is extends to $f$ but $M$ is duo therefore $\beta=\theta_{\left.\right|_{N}}: N \rightarrow N$ extends to $f$, thus $N$ is quasi-injective [2].

It is proved in [3], that every fully $R_{\Gamma}$-idempotent $R_{\Gamma}$-module is duo.

Corollary 3.12. If $M$ is fully $R_{\Gamma}$-idempotent $I \Gamma$-Extending $\quad R_{\Gamma}$-module, then every $R_{\Gamma}$-submodule of $M$ is quasi-injective.

We need the following lemma to prove Proposition(3.14).

Lemma 3.13. Let $M$ be an $R_{\Gamma}$-module. If $A$ essential $R_{\Gamma}$-submodule of $M$ and $B$ is a closed $R_{\Gamma}$-submodule of $M$, then $A \cap B$ is closed in $A$.
Mehdi .S / Saad.A / Emad.A

Proof: Let $B$ be a closed $R_{\Gamma}$-submodule of $M$ and $A$ essential $R_{\Gamma}$-submodule of $M$. By Lemma(3.5) in [2] $B$ must be a complement of some $R_{\Gamma}$-submodule $T$ of $M,(B \cap A) \cap(T \cap A)$ $=(B \cap T) \cap A=0$. Assume there is an $R_{\Gamma}$-submodule $N$ of $A$ contains $B \cap A$ properly, then $(B+N) \cap T \neq 0$, so there exists $0 \neq t=b+n$ where $t \in T, b \in B$ and $n \in N$, since $A$ essential in $M$, then there exists $r_{1}, r_{2}, \ldots, r_{n} \in R$ and $\gamma_{1}, \gamma_{2}, \ldots, \gamma_{n} \in \Gamma$ such that $0 \neq \sum_{i=1}^{n} r_{i} \gamma_{i} t=\sum_{i=1}^{n} r_{i} \gamma_{i} b+\sum_{i=1}^{n} r_{i} \gamma_{i} n \in$ $T \cap A$, so $\sum_{i=1}^{n} r_{i} \gamma_{i} b=\sum_{i=1}^{n} r_{i} \gamma_{i} t-\sum_{i=1}^{n} r_{i} \gamma_{i} n$ $\in B \cap A \subseteq N, \quad$ hence $\quad 0 \neq \sum_{i=1}^{n} r_{i} \gamma_{i} t \in$ $(T \cap A) \cap N, \quad$ so $B \cap A$ is maximal $R_{\Gamma}$-submodule of $A$ with respect to $(B \cap A) \cap(T \cap A)=0$, hence $B \cap A$ is a complement of $T \cap A$ in $A$.

Proposition 3.14. Let $M$ be a $Q I \Gamma$-Extending $R_{\Gamma}$-module and $N$ be a nontrivial closed $R_{\Gamma}$-submodule of $M$. Then $N$ and $N^{c}$ are quasi-injective $R_{\Gamma}$-submodule of $M$.

Proof: Let $N$ be a nontrivial closed $R_{\Gamma}$-submodule of an $Q I \Gamma$-Extending $R_{\Gamma}$-module $M$. Then $N$ is a quasi-injective by Proposition(3.2). In case $M=N \oplus N^{c}$, then $N^{c}$ is a proper closed in $M$ and hence $N^{c}$ is a quasi-injective by Proposition(3.2), in case $M \neq N \oplus N^{c}$, then there is a quasi-injective $R_{\Gamma}$-submodule $Q$ of $M$ such that $N \oplus$ $N^{c} \leq_{e} Q \leq M \quad$ since $\quad N \oplus N^{c} \leq_{e} M \quad$ by Lemma(3.4) in [2], then $Q \leq_{e} M$ but $N$ is closed in $M$, so $N=N \cap Q$ is closed in $Q$ by Lemma(3.13), hence $N$ is a quasi-injective by Corollary(3.11) in [2].

Direct sum of two QI - Extending need not be QI -Extending, for example see Examples(3.8)(6). 
Proposition 3.15. If the direct sum of every two $Q I \Gamma$-Extending is $Q I \Gamma$-Extending, then $M$ is quasi-injective if and only if $M$ is $Q I \Gamma-$ Extending.

Proof: Let $M$ be $Q I \Gamma$-Extending, since $Q(M)$ is quasi-injective, so $Q(M)$ is $Q I \Gamma$-Extending, hence $M \oplus Q(M)$ is $Q I \Gamma$-Extending, by Proposition(3.2) $M$ is quasi-injective.

\section{References}

1. M. S. Abbas, S. A. Al-Saadi, E. A. Shallal, (2016), Injective Gamma Modules, Annals of Pure and Applied Mathematics, 12(1), 85-94.

2. M. S. Abbas, S. A. Al-Saadi, E. A. Shallal, (2016), Quasi-Injective Gamma Modules, Int. J. of Advanced Research, 4(10), 327333.
3. M. S. Abbas, S. A. Al-Saadi, E. A. Shallal, (2017), Some Generalizations of Semisimple Gamma Rings, Journal of Science, 2017, 58(3C), 1720-1728.

4. R. Ameri and R.Sadeghi, (2010), Gamma Modules, Ratio Mathematica, 20, 127-147.

5. W. E. Barnes,(1966), On The $\Gamma$-Ring of Nobusawa, Pacific Journal of Mathematics, 18(3), 411-422.

6. S. Kyuno,(1975), On the radicals of $\Gamma$-Rings, Osaka J. Math. , 12, 639-645.

7. N. Nobusawa , (1964), On a Generalization of the ring theory, Osaka Journal Math., 1, 81-89.

8. S. Mohammed, B. J. Muller, (1990), Continuous and discrete modules, Cambridge Univ. Prees. , New York.

9. S. Uddin, S. Islam, (2012), Semi-Prime Ideals of Gamma Rings, Annals of Pure and Applied Mathematics, 1(2), 186-191.

\section{مقاسات التوسع (شبهه - ) الاغمارية من نمط كاما}

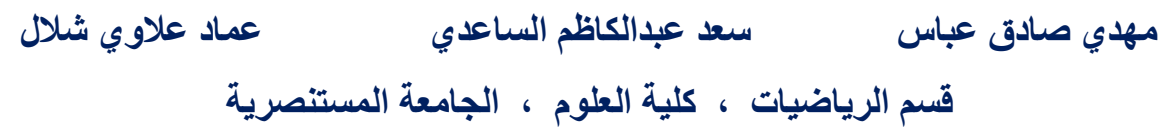

